Clinical Medicine: Case Report

Poster

Abstract ID: 45

\title{
Common Iliac Artery Aneurysm: Management Issues In The Era Of Endovascular Treatment
}

\author{
M Salehudin C.Z $Z^{\mathrm{a}} \mid$ Iqramie M.L ${ }^{\mathrm{a}} \mid$ Ahmad Faidzal Othman ${ }^{\mathrm{b}}$ \\ ${ }^{a}$ Department of Surgery, Hospital Tengku Ampuan Afzan, Kuantan, Pahang, Malaysia \\ ${ }^{b}$ Department of Surgery, Kulliyyah of Medicine, International Islamic University Malaysia
}

Common iliac artery aneurysm (CIAA) is rare. It only constitutes about $2 \%$ of all abdominal aneurysms. CIAA develops silently with typical presentation of hemorrhagic shock after rupture, which carries a very high perioperative mortality. Atypical presentation includes unilateral lower limb weakness, pain and swelling. These symptoms should alert clinicians to the differential diagnosis of iliac artery aneurysm. Early detection and investigations are paramount since immediate intervention can considerably improve the outcome. We will highlight 3 cases of symptomatic common iliac artery aneurysm with different treatment modalities. The options of management, including literature review regarding open and endovascular treatment will be highlighted.

KEYWORDS: Iliac artery aneurysm, open surgery, endovascular treatment 